\title{
EFFECT OF THERMAL STRESSES AND REDUCED DIFFERENTIAL TRANSFORM ON BENDING OF THE RECTANGULAR PLATE
}

\author{
Chandrashekhar S. Sutar ${ }^{1}$, And Sandip N. Patil ${ }^{2}$ \\ ${ }^{1}$ Department of Mathematics and Statistics, PSGVP Mandals Arts, Science \\ and Commerce College, Shahada, India, sutarchandu@gmail.com \\ ${ }^{2}$ N.Y.N.C. Arts, Science and Commerce College, Chalisgaon, India, \\ snpatil2007@gmail.com
}

\begin{abstract}
The bending of the rectangular plate exists for a large length comparing with its width as we have considered the simply supported plate. Also, it gets the accuracy, if the plate is attached between enough distance from the ends. This paper concern with the study of bending moment in the rectangular plate by using the thermal stresses. In this paper, we have considered the mathematical model of the unsteady state three-dimensional heat conduction equation for a thick rectangular plate with non-zero temperature initially. We have determined the thermal stresses and the bending moment in the rectangular plate by using the reduced differential transform method. The results are discussed by considering the special cases for copper material. Also, results are shown graphically. The graphs are drawn by using mathematical software Scilab.

Key words : Bending moment, Heat conduction, Reduced differential transform, Thermal stresses.
\end{abstract}

\section{INTRODUCTION}

Material's important properties are dependent on temperature. Thus temperature field acts as an important role in thermoelasticity. Nowacki [14] has studied the thermoelastic model in a circular plate subjected to an axisymmetric temperature distribution on the upper surface with zero temperature on the lower surface and with the circular edge thermally insulated. Noda et al. [13] have studied the fundamental theory of thermal stresses. Also, they have discussed the applications. Ozicik [15] in his book discussed the heat conduction in various solids. He has considered various examples of solids and determined the solutions. Recently Chaudhari and Sutar [4] analyzed the thermal stresses of two-dimensional rectangular plate with hyperbolic heat conduction. In their mathematical model they have used differential transform method to find the solution. Mansfield [9] discussed the elastic plate behavior and the bending and stretching of plates. Also, he has studied the deflection theory and thermal effects of various-shaped plates. Timoshenko and Woinowsky-Krieger [19] have studied bending of plates and described small deflections and pure bending of plates. Also they have analyzed the bending of plates depends on the thickness and the dimensions of the plate. Srivastava

2020 Mathematics Subject Classification:58J35, 35Q74,35K05.

Received: 10-12-2020, accepted: 06-04-2021. 
et al.[17] have discussed the reduced differential transform method. Deshmukh et al.[6] have considered the simply supported rectangular plate and determined the deflection.Yuemei and Rui [21] studied the bending analysis of a rectangular plate, where he considered the rectangular plate with two adjacent edges free and other edges are clamped. Boley and Weiner [2] explain the theory of thermal stresses for various solids. Jane and Hong [7] have studied the thermal bending analysis of orthotropic plates by the generalized differential quadrature method. Yos and Kraiwood [20] have given the bending behaviors of simply supported rectangular plates with an internal line sagged and unsagged supports. Cattaneo and Mehta [3],[10] have studied the heat conduction analysis. Barletta [1], Chen [5], Mallick [8], Morimoto [12] and Sherief [16] have discussed the thermoelastic behavior. Al-Amr [11] and Taha [18] gives the details of the reduced differential transform method.

In this paper, we have considered the thick rectangular plate which has simply supported edges with thickness ' $h$ ' and dimensions as $0 \leq x \leq a, 0 \leq y \leq b$, $0 \leq z \leq h$. The bending moment along direction ' $z$ ' has been determined by the reduced differential transform method.

\section{REDUCED DIFFERENTIAL TRANSFORM METHOD}

If $u(x, y, z, t)$ is analytic and continuosly differentiable with repect to $x, y, z, t$ then the reduced differential transform function of $u(x, y, z, t)$ is defined as Srivastava et al.[17],

$$
U_{k}(x, y, z, t)=\frac{1}{k !}\left[\frac{\partial^{k}}{\partial t^{k}} u(x, y, z, t)\right]_{t=t_{0}}
$$

The inverse differential reduced transformed function of $U_{k}(x, y, z, t)$ is given by Srivastava et al.[17],

$$
u(x, y, z, t)=\sum_{k=0}^{\infty} U_{k}(x, y, z, t)\left(t-t_{0}\right)^{k}
$$

\section{STATEMENT OF THE PROBLEM}

Consider the three dimensional rectangular plate with simply supported edges [Fig.1] with dimensions $0 \leq x \leq a, 0 \leq y \leq b, 0 \leq z \leq h$. The bending moment along direction ' $z$ ' and thickness ' $h$ '.

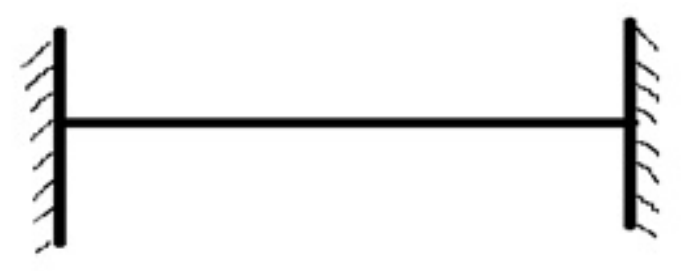

FiguRE 1. Rectangular plate with simply supported edges

The heat conduction equation in three dimensions, Ozicik [15] is given by

$$
\frac{\partial^{2} T}{\partial x^{2}}+\frac{\partial^{2} T}{\partial y^{2}}+\frac{\partial^{2} T}{\partial z^{2}}=\frac{1}{\alpha} \frac{\partial T}{\partial t}
$$


where $\alpha=$ Thermal diffusivity of material, with initial condition

$$
[T(x, y, z, t)]_{t=0}=a_{0}(x, y, z)
$$

The stress function $\chi$ is given by the equation, Noda et al. [13],

$$
\chi=\chi_{c}+\chi_{p}
$$

Where the $\chi_{c}$ is complementary function and $\chi_{p}$ is the particular solution of $\chi$. The $\chi_{c}$ satisfies the following equation, Noda et al. [13],

$$
\left(\frac{\partial^{2}}{\partial x^{2}}+\frac{\partial^{2}}{\partial y^{2}}+\frac{\partial^{2}}{\partial z^{2}}\right)\left(\frac{\partial^{2} \chi_{c}}{\partial x^{2}}+\frac{\partial^{2} \chi_{c}}{\partial y^{2}}+\frac{\partial^{2} \chi_{c}}{\partial z^{2}}\right)=0
$$

and $\chi_{p}$ is obtained from the equation, Noda et al. [13],

$$
\frac{\partial^{2} \chi_{p}}{\partial x^{2}}+\frac{\partial^{2} \chi_{p}}{\partial y^{2}}+\frac{\partial^{2} \chi_{p}}{\partial z^{2}}=-\alpha E \Gamma
$$

where $\Gamma=T-[T]_{t=0}=b$ (let).

$E=$ Young's Modulus of the material.

The thermal stresses are given by the equations, Noda et al. [13],

$$
\sigma_{x x}=\frac{\partial^{2} \chi}{\partial y^{2}}, \sigma_{y y}=\frac{\partial^{2} \chi}{\partial x^{2}}, \sigma_{x y}=-\frac{\partial^{2} \chi}{\partial x \partial y}
$$

The bending moment regarding thermal stresses in $z$ direction are given by the equation

$$
M_{x}=\int_{\frac{-h}{2}}^{\frac{h}{2}} \sigma_{x x} z d z, M_{y}=\int_{\frac{-h}{2}}^{\frac{h}{2}} \sigma_{y y} z d z, M_{x y}=\int_{\frac{-h}{2}}^{\frac{h}{2}} \sigma_{x y} z d z .
$$

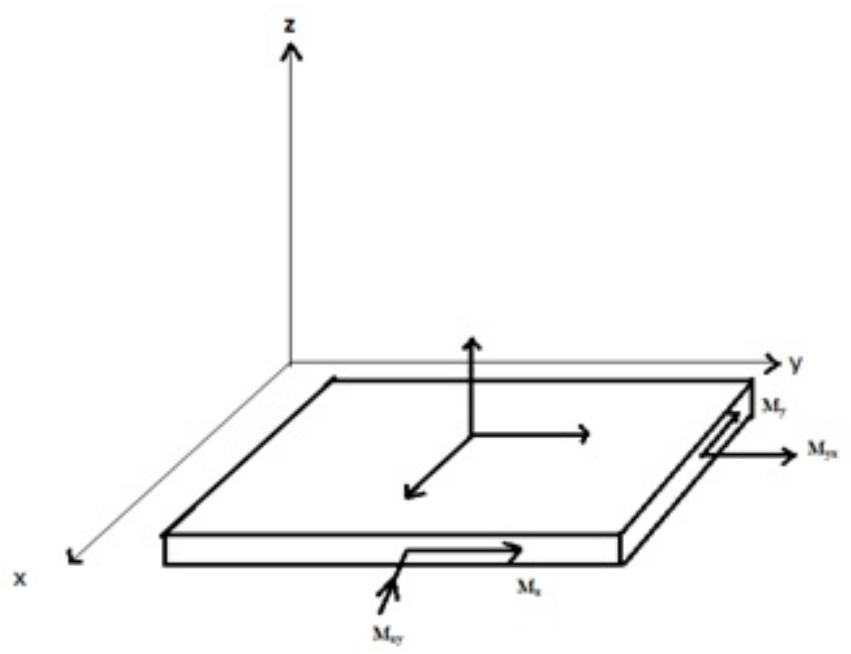

FIGURE 2. Direction of bending moments 


\section{SOLUTION OF THE PROBLEM}

Applying Reduced differential transform to equation (3) and (4) we get

$$
\begin{gathered}
(k+1) T_{k+1}(x, y, z)=\alpha\left[\frac{\partial^{2}}{\partial x^{2}} T_{k}(x, y, z)+\frac{\partial^{2}}{\partial y^{2}} T_{k}(x, y, z)+\frac{\partial^{2}}{\partial z^{2}} T_{k}(x, y, z)\right] \\
T_{0}(x, y, z)=a_{0}(x, y, z)
\end{gathered}
$$

Using (11) in (10) we get,

$$
T_{k}(x, y, z)=\frac{\alpha^{k}}{k !} a_{k}
$$

where $a_{k}=\left(a_{k-1}\right)_{x x}+\left(a_{k-1}\right)_{y y}+\left(a_{k-1}\right)_{z z}, k=1,2,3 \ldots$

Applying inverse reduced differential transform [(Equation 2)] we get

$$
T(x, y, z, t)=a_{0}(x, y, z)+\sum_{k=1}^{\infty} \frac{\alpha^{k}}{k !} a_{k} t^{k} .
$$

\section{DETERMINATION OF STRESS FUNCTION}

Assume that the $\chi_{c}$, which satisfies the equation (6) as

$$
\begin{aligned}
& \chi_{c}=f_{0}+f_{1} x+f_{2} x^{2}+f_{3} x^{3}-\frac{1}{24}\left[\left(p_{0}\right)_{y y}+\left(p_{0}\right)_{z z}+2\left(f_{2}\right)_{y y}+2\left(f_{2}\right)_{z z}\right] x^{4} \\
& -\frac{1}{120}\left[\left(p_{1}\right)_{y y}+\left(p_{1}\right)_{z z}+6\left(f_{3}\right)_{y y}+6\left(f_{3}\right)_{z z}\right] x^{5}+\sum_{k=6}^{\infty} \frac{f_{k}}{k(k-1)(k-2)(k-3)} x^{k},
\end{aligned}
$$

where, at $x=0, \chi_{c}=f_{0}$,

$$
\frac{\partial \chi_{c}}{\partial x}=f_{1}(y, z), \frac{\partial^{2} \chi_{c}}{\partial x^{2}}=2 f_{2}(y, z), \frac{\partial^{3} \chi_{c}}{\partial x^{3}}=6 f_{3}(y, z),
$$

and $f_{k}=-\left(p_{k-4}\right)_{y y}-\left(p_{k-4}\right)_{z z}-(k-4)(k-5)\left[\left(f_{k-2}\right)_{y y}+\left(f_{k-2}\right)_{z z}\right]$,

$$
\begin{aligned}
& f_{4}=\left(p_{0}\right)_{y y}+\left(p_{0}\right)_{z z}+2\left(f_{2}\right)_{y y}+2\left(f_{2}\right)_{z z}, \\
& f_{5}=\left(p_{1}\right)_{y y}+\left(p_{1}\right)_{z z}+6\left(f_{3}\right)_{y y}+6\left(f_{3}\right)_{z z}, \\
& p_{k}=(k+1)(k+2)\left(\chi_{c}\right)_{k+2}+\frac{\partial^{2}}{\partial y^{2}}\left(\chi_{c}\right)_{k}+\frac{\partial^{2}}{\partial z^{2}}\left(\chi_{c}\right)_{k} .
\end{aligned}
$$

Also assume that the $\chi_{p}$ which satisfies the equation (7)as

$$
\begin{aligned}
\chi_{p}=-\frac{\alpha E}{2} b_{0} x^{2} & -\frac{\alpha E}{6} b_{1} x^{3}+\frac{1}{12}\left\{-\alpha E b_{2}+\frac{\alpha E}{2}\left[\left(b_{0}\right)_{y y}+\left(b_{0}\right)_{z z}\right]\right\} x^{4} \\
& +\frac{1}{20}\left\{-\alpha E b_{3}+\frac{\alpha E}{6}\left[\left(b_{1}\right)_{y y}+\left(b_{1}\right)_{z z}\right]\right\} x^{5}+\sum_{k=6}^{\infty} \frac{q_{k}}{k(k-1)} x^{k},
\end{aligned}
$$

where $\chi_{p}=0$ at $x=0$,

$$
\begin{aligned}
& \frac{\partial \chi_{p}}{\partial x}=0 \text { at } x=0 \\
& q_{k}=-\alpha E b_{k-2}-\frac{1}{(k-2)(k-3)}\left[\left(q_{k-2}\right)_{y y}+\left(q_{k-2}\right)_{z z}\right], \\
& q_{4}=-\alpha E b_{2}+\frac{\alpha E}{2}\left[\left(b_{0}\right)_{y y}+\left(b_{0}\right)_{z z}\right],
\end{aligned}
$$




$$
q_{5}=-\alpha E b_{3}+\frac{\alpha E}{6}\left[\left(b_{1}\right)_{y y}+\left(b_{1}\right)_{z z}\right] .
$$

From equations (5),(14) and (16) we get,

$$
\begin{aligned}
& \chi=f_{0}+f_{1} x+ f_{2} x^{2}+f_{3} x^{3}-\frac{1}{24}\left[\left(p_{0}\right)_{y y}+\left(p_{0}\right)_{z z}+2\left(f_{2}\right)_{y y}+2\left(f_{2}\right)_{z z}\right] x^{4} \\
&-\frac{1}{120}\left[\left(p_{1}\right)_{y y}+\right.\left.\left(p_{1}\right)_{z z}+6\left(f_{3}\right)_{y y}+6\left(f_{3}\right)_{z z}\right] x^{5}+\sum_{k=6}^{\infty} \frac{f_{k}}{k(k-1)(k-2)(k-3)} x^{k} \\
&-\frac{\alpha E}{2} b_{0} x^{2}-\frac{\alpha E}{6} b_{1} x^{3}+\frac{1}{12}\left\{-\alpha E b_{2}+\frac{\alpha E}{2}\left[\left(b_{0}\right)_{y y}+\left(b_{0}\right)_{z z}\right]\right\} x^{4} \\
&+\frac{1}{20}\left\{-\alpha E b_{3}+\frac{\alpha E}{6}\left[\left(b_{1}\right)_{y y}+\left(b_{1}\right)_{z z}\right]\right\} x^{5}+\sum_{k=6}^{\infty} \frac{q_{k}}{k(k-1)} x^{k} .
\end{aligned}
$$

\section{DETERMINATION OF THERMAL STRESS}

The thermal stresses are given by the equation (8) and (17)

$$
\begin{gathered}
\sigma_{x x}=\left(f_{0}\right)_{y y}+x\left(f_{1}\right)_{y y}+x^{2}\left(f_{2}\right)_{y y}+x^{3}\left(f_{3}\right)_{y y}-\left\{\frac{1}{24}\left[\left(p_{0}\right)_{y y}+\left(p_{0}\right)_{z z}+2\left(f_{2}\right)_{y y}+2\left(f_{2}\right)_{z z}\right]\right\}_{y y} x^{4} \\
-\left\{\frac{1}{120}\left[\left(p_{1}\right)_{y y}+\left(p_{1}\right)_{z z}+6\left(f_{3}\right)_{y y}+6\left(f_{3}\right)_{z z}\right]\right\}_{y y} x^{5}+\sum_{k=6}^{\infty} \frac{\left(f_{k}\right)_{y y}}{k(k-1)(k-2)(k-3)} x^{k} \\
-\frac{\alpha E}{2}\left(b_{0}\right)_{y y} x^{2}-\frac{\alpha E}{6}\left(b_{1}\right)_{y y} x^{3}+\frac{1}{12}\left\{-\alpha E\left(b_{2}\right)_{y y}+\frac{\alpha E}{2}\left[\left(b_{0}\right)_{y y}+\left(b_{0}\right)_{z z}\right]_{y y}\right\} x^{4} \\
+\frac{1}{20}\left\{-\alpha E\left(b_{3}\right)_{y y}+\frac{\alpha E}{6}\left[\left(b_{1}\right)_{y y}+\left(b_{1}\right)_{z z}\right]_{y y}\right\} x^{5}+\sum_{k=6}^{\infty} \frac{\left(q_{k}\right)_{y y}}{k(k-1)} x^{k} .
\end{gathered}
$$

$$
\begin{aligned}
& \sigma_{y y}=\left(f_{0}\right)_{x x}+\left(f_{1}\right)_{x x} x+2 f_{2}+\left(f_{2}\right)_{x x} x^{2}+6 f_{3} x+\left(f_{3}\right)_{x x} x^{3} \\
& -\frac{1}{24}\left\{\left[\left(p_{0}\right)_{y y}+\left(p_{0}\right)_{z z}+2\left(f_{2}\right)_{y y}+2\left(f_{2}\right)_{z z}\right]\right\}_{x x} x^{4}-\frac{1}{2}\left[\left(p_{0}\right)_{y y}+\left(p_{0}\right)_{z z}+2\left(f_{2}\right)_{y y}+2\left(f_{2}\right)_{z z}\right] x^{2} \\
& -\frac{1}{120}\left\{\left[\left(p_{1}\right)_{y y}+\left(p_{1}\right)_{z z}+6\left(f_{3}\right)_{y y}+6\left(f_{3}\right)_{z z}\right]\right\}_{x x} x^{5}-\frac{1}{6}\left[\left(p_{1}\right)_{y y}+\left(p_{1}\right)_{z z}+6\left(f_{3}\right)_{y y}+6\left(f_{3}\right)_{z z}\right] x^{3} \\
& \quad+\sum_{k=6}^{\infty}\left\{\frac{\left(f_{k}\right)_{x x}}{k(k-1)(k-2)(k-3)} x^{k}+\frac{f_{k} k(k-1)}{k(k-1)(k-2)(k-3)} x^{k-2}\right\} \\
& -\frac{\alpha E}{2}\left(\left(b_{0}\right)_{x x} x^{2}+2 b_{0}\right)-\frac{\alpha E}{6}\left(\left(b_{1}\right)_{x x} x^{3}+6 x b_{1}\right)+\left\{-\alpha E b_{2}+\frac{\alpha E}{2}\left[\left(b_{0}\right)_{y y}+\left(b_{0}\right)_{z z}\right]\right\} x^{2} \\
& +\frac{1}{12}\left\{-\alpha E\left(b_{2}\right)_{x x}+\frac{\alpha E}{2}\left[\left(b_{0}\right)_{y y}+\left(b_{0}\right)_{z z}\right]_{x x}\right\} x^{4}+\left\{-\alpha E b_{3}+\frac{\alpha E}{6}\left[\left(b_{1}\right)_{y y}+\left(b_{1}\right)_{z z}\right]\right\} x^{3} \\
& +\frac{1}{20}\left\{-\alpha E\left(b_{3}\right)_{x x}+\frac{\alpha E}{6}\left[\left(b_{1}\right)_{y y}+\left(b_{1}\right)_{z z}\right]_{x x}\right\} x^{5}+\sum_{k=6}^{\infty}\left\{\frac{k(k-1) q_{k}}{k(k-1)} x^{k-2}+\frac{\left(q_{k}\right)_{x x}}{k(k-1)} x^{k}\right\} .
\end{aligned}
$$




$$
\begin{aligned}
& \sigma_{x y}=-\left(f_{0}\right)_{x y}-x\left(f_{1}\right)_{x y}-2 x\left(f_{2}\right)_{y}-x^{2}\left(f_{2}\right)_{x y}-3 x^{2}\left(f_{3}\right)_{y}-\left(f_{3}\right)_{x y} x^{3} \\
+ & \frac{1}{24}\left\{\left[\left(p_{0}\right)_{y y}+\left(p_{0}\right)_{z z}+2\left(f_{2}\right)_{y y}+2\left(f_{2}\right)_{z z}\right]\right\}_{x y} x^{4}+\frac{1}{6}\left[\left(p_{0}\right)_{y y}+\left(p_{0}\right)_{z z}+2\left(f_{2}\right)_{y y}+2\left(f_{2}\right)_{z z}\right]_{y} x^{3} \\
- & \frac{1}{120}\left\{\left[\left(p_{1}\right)_{y y}+\left(p_{1}\right)_{z z}+6\left(f_{3}\right)_{y y}+6\left(f_{3}\right)_{z z}\right]\right\}_{x y} x^{5}-\frac{1}{24}\left[\left(p_{1}\right)_{y y}+\left(p_{1}\right)_{z z}+6\left(f_{3}\right)_{y y}+6\left(f_{3}\right)_{z z}\right]_{y} x^{4} \\
& -\sum_{k=6}^{\infty}\left\{\frac{\left(f_{k}\right)_{x y}}{k(k-1)(k-2)(k-3)} x^{k}+\frac{\left(f_{k}\right)_{y}}{(k-1)(k-2)(k-3)} x^{k}\right\} \\
+ & \frac{\alpha E}{2}\left(\left(b_{0}\right)_{x y} x^{2}+2\left(b_{0}\right)_{y} x\right)+\frac{\alpha E}{6}\left(\left(b_{1}\right)_{x y} x^{3}+3 x^{2}\left(b_{1}\right)_{y}\right)-\frac{1}{12}\left\{-\alpha E b_{2}+\frac{\alpha E}{2}\left[\left(b_{0}\right)_{y y}+\left(b_{0}\right)_{z z}\right]\right\}_{x y} x^{4} \\
- & \frac{1}{3}\left\{-\alpha E b_{2}+\frac{\alpha E}{2}\left[\left(b_{0}\right)_{y y}+\left(b_{0}\right)_{z z}\right]\right\}_{y} x^{3}-\frac{1}{20}\left\{-\alpha E b_{3}+\frac{\alpha E}{6}\left[\left(b_{1}\right)_{y y}+\left(b_{1}\right)_{z z}\right]\right\}_{x y} x^{5} \\
- & \frac{1}{4}\left\{-\alpha E b_{3}+\frac{\alpha E}{6}\left[\left(b_{1}\right)_{y y}+\left(b_{1}\right)_{z z}\right]\right\}_{y} x^{4}-\sum_{k=6}^{\infty}\left\{\frac{\left(q_{k}\right)_{x y}}{k(k-1)} x^{k}+\frac{\left(q_{k}\right)_{y}}{(k-1)} x^{k-1}\right\} . \quad
\end{aligned}
$$

\section{DETERMINATION OF BENDING MOMENT}

The bending moments of rectangular plate in ' $z$ ' direction by using equation (9) and equations (18) to (20) are given by

$$
\begin{gathered}
M_{x}=\int_{\frac{-h}{2}}^{\frac{h}{2}}\left\{\left(f_{0}\right)_{y y}+x\left(f_{1}\right)_{y y}+x^{2}\left(f_{2}\right)_{y y}+x^{3}\left(f_{3}\right)_{y y}\right. \\
-\left\{\frac{1}{24}\left[\left(p_{0}\right)_{y y}+\left(p_{0}\right)_{z z}+2\left(f_{2}\right)_{y y}+2\left(f_{2}\right)_{z z}\right]\right\}_{y y} x^{4} \\
-\left\{\frac{1}{120}\left[\left(p_{1}\right)_{y y}+\left(p_{1}\right)_{z z}+6\left(f_{3}\right)_{y y}+6\left(f_{3}\right)_{z z}\right]\right\}_{y y} x^{5} \\
+\sum_{k=6}^{\infty} \frac{\left(f_{k}\right)_{y y}}{k(k-1)(k-2)(k-3)} x^{k} \\
-\frac{\alpha E}{2}\left(b_{0}\right)_{y y} x^{2}-\frac{\alpha E}{6}\left(b_{1}\right)_{y y} x^{3}+\frac{1}{12}\left\{-\alpha E\left(b_{2}\right)_{y y}+\frac{\alpha E}{2}\left[\left(b_{0}\right)_{y y}+\left(b_{0}\right)_{z z}\right]_{y y}\right\} x^{4} \\
\left.+\frac{1}{20}\left\{-\alpha E\left(b_{3}\right)_{y y}+\frac{\alpha E}{6}\left[\left(b_{1}\right)_{y y}+\left(b_{1}\right)_{z z}\right]_{y y}\right\} x^{5}+\sum_{k=6}^{\infty} \frac{\left(q_{k}\right)_{y y}}{k(k-1)} x^{k}\right\} z d z .
\end{gathered}
$$




$$
\begin{aligned}
& M_{y}=\int_{\frac{-h}{2}}^{\frac{h}{2}}\left\{\left(f_{0}\right)_{x x}+\left(f_{1}\right)_{x x} x+2 f_{2}+\left(f_{2}\right)_{x x} x^{2}+6 f_{3} x+\left(f_{3}\right)_{x x} x^{3}\right. \\
& -\frac{1}{24}\left\{\left[\left(p_{0}\right)_{y y}+\left(p_{0}\right)_{z z}+2\left(f_{2}\right)_{y y}+2\left(f_{2}\right)_{z z}\right]\right\}_{x x} x^{4}-\frac{1}{2}\left[\left(p_{0}\right)_{y y}+\left(p_{0}\right)_{z z}+2\left(f_{2}\right)_{y y}+2\left(f_{2}\right)_{z z}\right] x^{2} \\
& -\frac{1}{120}\left\{\left[\left(p_{1}\right)_{y y}+\left(p_{1}\right)_{z z}+6\left(f_{3}\right)_{y y}+6\left(f_{3}\right)_{z z}\right]\right\}_{x x} x^{5}-\frac{1}{6}\left[\left(p_{1}\right)_{y y}+\left(p_{1}\right)_{z z}+6\left(f_{3}\right)_{y y}+6\left(f_{3}\right)_{z z}\right] x^{3} \\
& +\sum_{k=6}^{\infty}\left\{\frac{\left(f_{k}\right)_{x x}}{k(k-1)(k-2)(k-3)} x^{k}+\frac{f_{k} k(k-1)}{k(k-1)(k-2)(k-3)} x^{k-2}\right\} \\
& -\frac{\alpha E}{2}\left(\left(b_{0}\right)_{x x} x^{2}+2 b_{0}\right)-\frac{\alpha E}{6}\left(\left(b_{1}\right)_{x x} x^{3}+6 x b_{1}\right)+\left\{-\alpha E b_{2}+\frac{\alpha E}{2}\left[\left(b_{0}\right)_{y y}+\left(b_{0}\right)_{z z}\right]\right\} x^{2} \\
& +\frac{1}{12}\left\{-\alpha E\left(b_{2}\right)_{x x}+\frac{\alpha E}{2}\left[\left(b_{0}\right)_{y y}+\left(b_{0}\right)_{z z}\right]_{x x}\right\} x^{4}+\left\{-\alpha E b_{3}+\frac{\alpha E}{6}\left[\left(b_{1}\right)_{y y}+\left(b_{1}\right)_{z z}\right]\right\} x^{3} \\
& \left.+\frac{1}{20}\left\{-\alpha E\left(b_{3}\right)_{x x}+\frac{\alpha E}{6}\left[\left(b_{1}\right)_{y y}+\left(b_{1}\right)_{z z}\right]_{x x}\right\} x^{5}+\sum_{k=6}^{\infty}\left\{\frac{k(k-1) q_{k}}{k(k-1)} x^{k-2}+\frac{\left(q_{k}\right)_{x x}}{k(k-1)} x^{k}\right\}\right\} z d z . \\
& M_{x y}=\int_{\frac{-h}{2}}^{\frac{h}{2}}\left\{-\left(f_{0}\right)_{x y}-x\left(f_{1}\right)_{x y}-2 x\left(f_{2}\right)_{y}-x^{2}\left(f_{2}\right)_{x y}-3 x^{2}\left(f_{3}\right)_{y}-\left(f_{3}\right)_{x y} x^{3}\right. \\
& +\frac{1}{24}\left\{\left[\left(p_{0}\right)_{y y}+\left(p_{0}\right)_{z z}+2\left(f_{2}\right)_{y y}+2\left(f_{2}\right)_{z z}\right]\right\}_{x y} x^{4}+\frac{1}{6}\left[\left(p_{0}\right)_{y y}+\left(p_{0}\right)_{z z}+2\left(f_{2}\right)_{y y}+2\left(f_{2}\right)_{z z}\right]_{y} x^{3} \\
& -\frac{1}{120}\left\{\left[\left(p_{1}\right)_{y y}+\left(p_{1}\right)_{z z}+6\left(f_{3}\right)_{y y}+6\left(f_{3}\right)_{z z}\right]\right\}_{x y} x^{5}-\frac{1}{24}\left[\left(p_{1}\right)_{y y}+\left(p_{1}\right)_{z z}+6\left(f_{3}\right)_{y y}+6\left(f_{3}\right)_{z z}\right]_{y} x^{4} \\
& -\sum_{k=6}^{\infty}\left\{\frac{\left(f_{k}\right)_{x y}}{k(k-1)(k-2)(k-3)} x^{k}+\frac{\left(f_{k}\right)_{y}}{(k-1)(k-2)(k-3)} x^{k}\right\} \\
& +\frac{\alpha E}{2}\left(\left(b_{0}\right)_{x y} x^{2}+2\left(b_{0}\right)_{y} x\right)+\frac{\alpha E}{6}\left(\left(b_{1}\right)_{x y} x^{3}+3 x^{2}\left(b_{1}\right)_{y}\right)-\frac{1}{12}\left\{-\alpha E b_{2}+\frac{\alpha E}{2}\left[\left(b_{0}\right)_{y y}+\left(b_{0}\right)_{z z}\right]\right\}_{x y} x^{4} \\
& -\frac{1}{3}\left\{-\alpha E b_{2}+\frac{\alpha E}{2}\left[\left(b_{0}\right)_{y y}+\left(b_{0}\right)_{z z}\right]\right\}_{y} x^{3}-\frac{1}{20}\left\{-\alpha E b_{3}+\frac{\alpha E}{6}\left[\left(b_{1}\right)_{y y}+\left(b_{1}\right)_{z z}\right]\right\}_{x y} x^{5} \\
& \left.-\frac{1}{4}\left\{-\alpha E b_{3}+\frac{\alpha E}{6}\left[\left(b_{1}\right)_{y y}+\left(b_{1}\right)_{z z}\right]\right\}_{y} x^{4}-\sum_{k=6}^{\infty}\left\{\frac{\left(q_{k}\right)_{x y}}{k(k-1)} x^{k}+\frac{\left(q_{k}\right)_{y}}{(k-1)} x^{k-1}\right\}\right\} z d z \text {. }
\end{aligned}
$$

\section{SPECIAL CASES}

Here we consider the copper plate with thickness $h=4 m, t=1 \mathrm{sec}$ for the special case. All numerical values have been considered regarding the copper plate.

Consider some special functions which satisfies all the conditions in equation (4) and (15) as

$$
\begin{aligned}
& a_{0}(x, y, z)=x^{2} y^{2} z \\
& f_{0}=z^{6}-y^{6}+2 y^{3} z^{3} \\
& f_{1}=0=f_{2} \\
& f_{3}=2 y^{3}+2 z^{3} .
\end{aligned}
$$

From equation (14) and (16) we get, $\chi_{c}=z^{6}-y^{6}+2 y^{3} z^{3}+2 x^{3} y^{3}+2 x^{3} z^{3}+15 x^{4} y^{2}-6 x^{4} y z-15 z^{2} x^{4}-\frac{6}{5} x^{5} y-\frac{6}{5} x^{5} z$ 
$\chi_{p}=-\alpha E x^{2}\left\{\alpha y^{2} z t+2 \alpha^{2} z t^{2}\right\}$.

Hence from equation (5) we get,

$$
\begin{aligned}
& \chi=z^{6}-y^{6}+2 y^{3} z^{3}+2 x^{3} y^{3}+2 x^{3} z^{3}+15 x^{4} y^{2}-6 x^{4} y z-15 z^{2} x^{4} \\
& -\frac{6}{5} x^{5} y-\frac{6}{5} x^{5} z-\alpha E x^{2}\left\{\alpha y^{2} z t+2 \alpha^{2} z t^{2}\right\} .
\end{aligned}
$$

From equation (8) we get thermal stresses are

$$
\begin{aligned}
& \sigma_{x x}=-30 y^{4}+12 y z^{3}+12 x^{3} y+30 x^{4}-2 \alpha^{2} z E x^{2} t \\
& \sigma_{y y}=12 x y^{3}+12 x z^{3}+180 x^{2} y^{2}-72 x^{2} y z-180 z^{2} x^{2}-24 x^{3} y-24 x^{3} z \\
& -\alpha E\left\{2 \alpha y^{2} z t+4 \alpha^{2} z t^{2}\right\} \\
& \sigma_{x y}=-18 x^{2} y^{2}-120 x^{3} y+6 x^{4}+24 x^{3} y z+4 \alpha^{2} \text { Exyzt. }
\end{aligned}
$$

From equation (9) we get the bending moments as

$$
\begin{aligned}
& M_{x}=\frac{768 y}{5}-\frac{32 \alpha^{2} E x^{2} t}{3} \\
& M_{y}=\frac{768 x}{5}-384 x^{2} y-128 x^{3}-\frac{16 \alpha E}{3}\left\{2 \alpha y^{2} t+4 \alpha^{2} t^{2}\right\} M_{x y}=128 x^{3} y+
\end{aligned}
$$
$\frac{64 \alpha^{2} \text { Exyt }}{3}$.

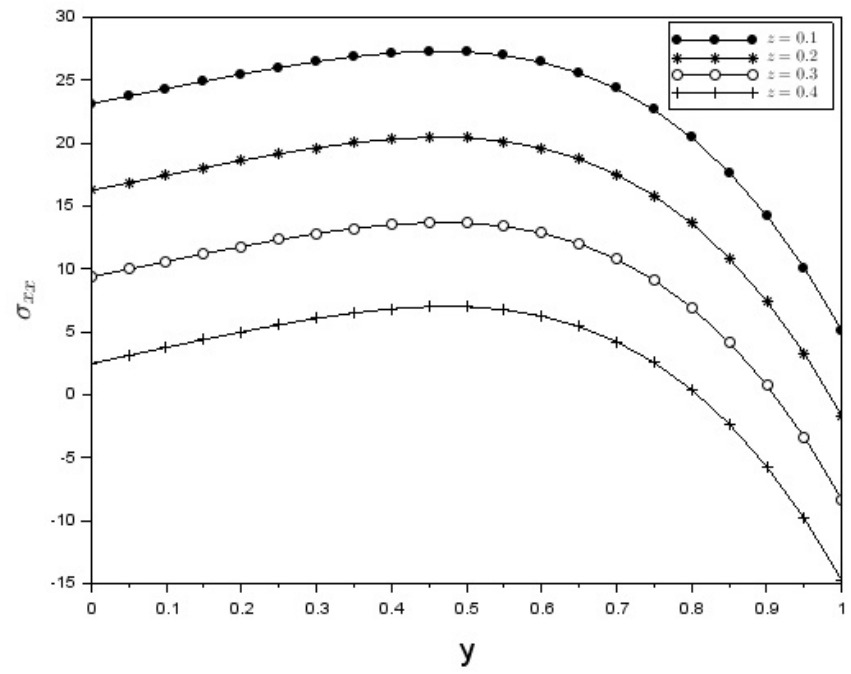

Figure 3. $\sigma_{x x} \mathrm{Vs}^{\prime} y^{\prime}$ with variation in ${ }^{\prime} z^{\prime}$ 


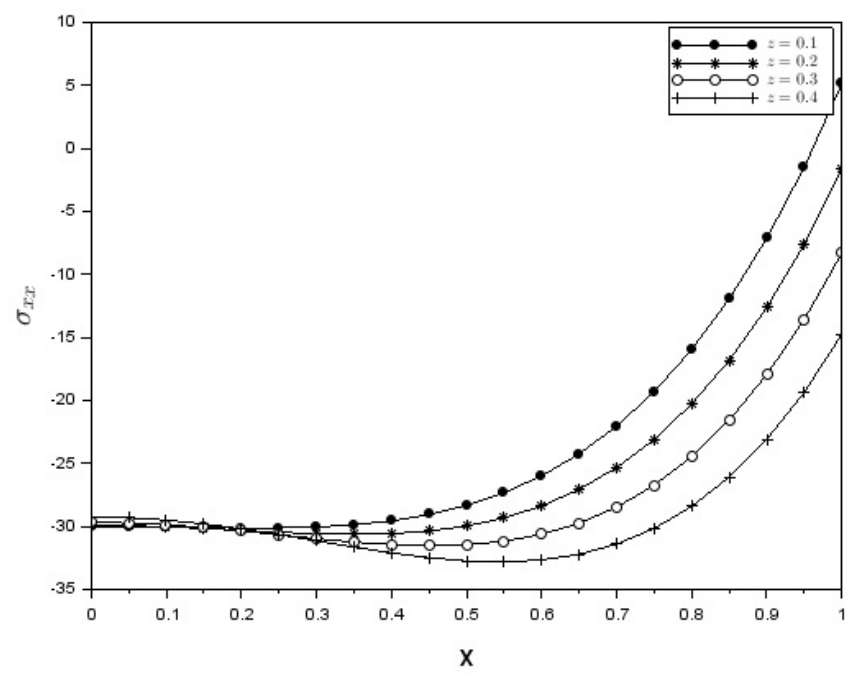

FIGURE $4 . \sigma_{x x} \operatorname{Vs}^{\prime} x^{\prime}$ with variation in ' $z^{\prime}$

Fig-3 and Fig-4 gives the relations of thermal stresses along with breadth $(y)$ and length $(x)$ respectively for given changes in heights. Whenever changes in breadth the stress decreases suddenly and an increase in length implies the sudden increase in stress.

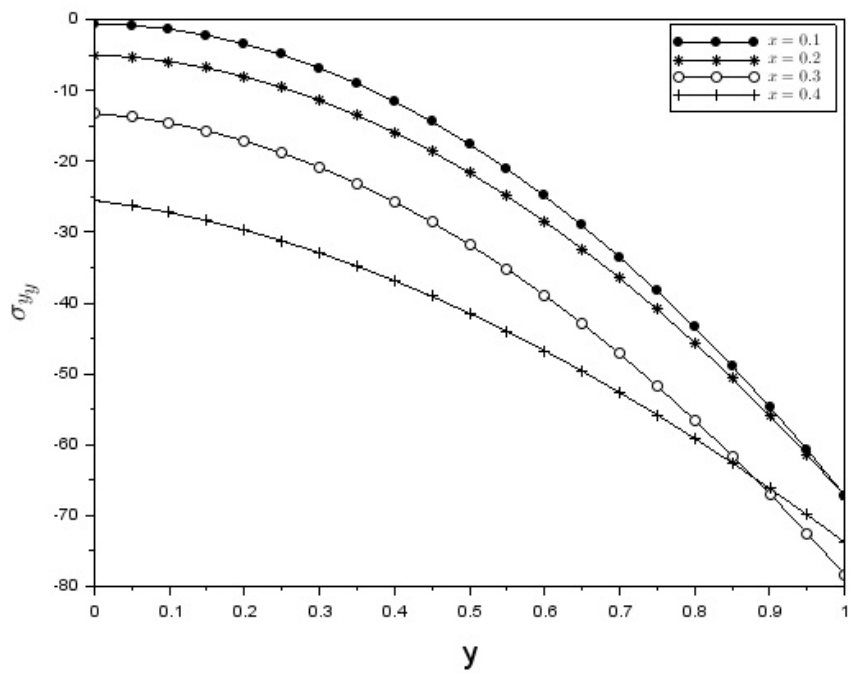

Figure 5. $\sigma_{y y} \mathrm{Vs}^{\prime} y^{\prime}$ with variation in ' $x$ '

Fig-5 explains the nature of thermal stress along with the breadth of the plate for the variations in length of the plate at constant heights. For the change in breadth of the plate there is a sudden change in stress and the intersection point sometime indicates that whenever there is not much changes in length, the thermal 
stresses remains constant.

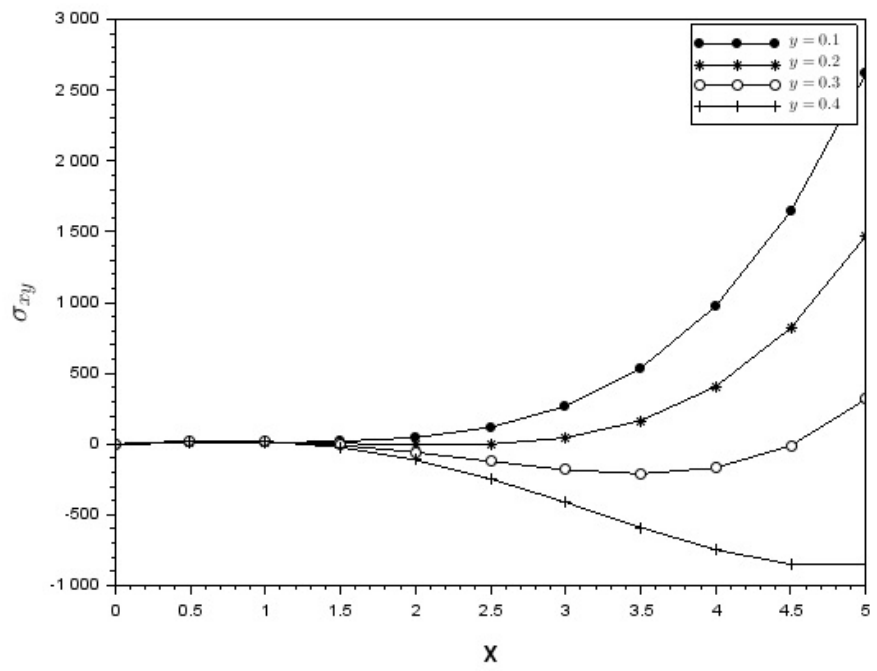

FiguRE 6. $\sigma_{x y} \mathrm{Vs}^{\prime} y^{\prime}$ with variation in ' $x^{\prime}$

Fig-6 gives the behavior of thermal stress with respective length at constant heights for changes in breadth.

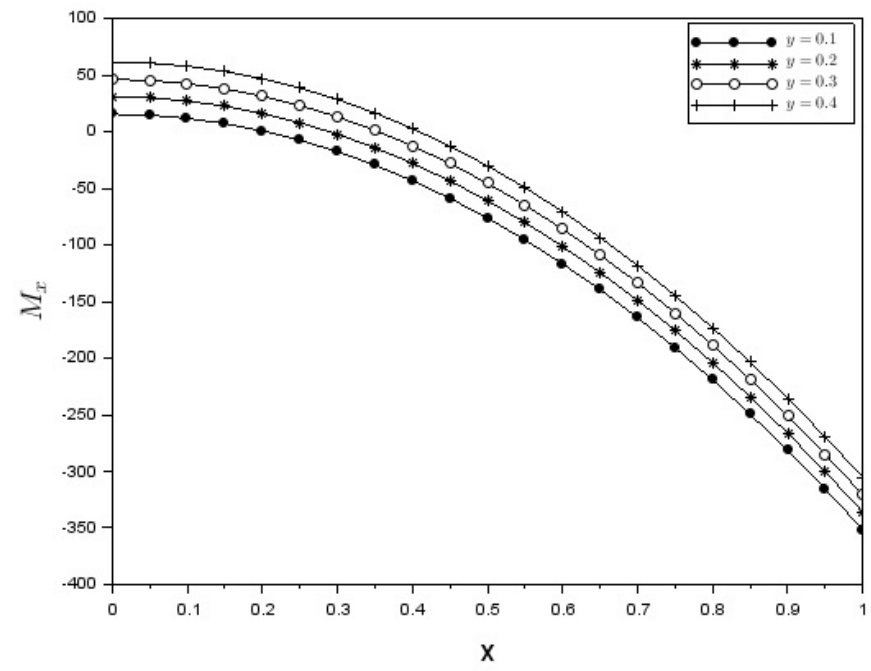

FiguRE 7. $M_{x} \mathrm{Vs}^{\prime} x^{\prime}$ with variation in ' $y$ '

Fig-7 explains the nature of bending moments with the length of the plate and the variations in breadth. The above figures explain the result that the bending 
moment decreases along the length.

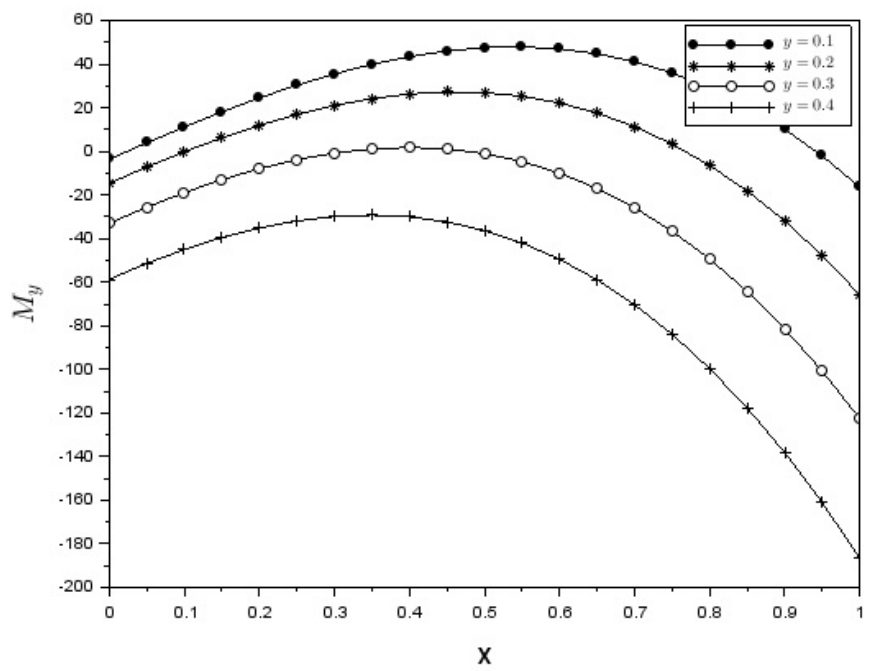

FiguRE 8. $M_{y} \mathrm{Vs}^{\prime} x^{\prime}$ with variation in ' $y$ '

Fig- 8 gives the variation of bending moments of the plate along the $z$-direction. The above figures explain the result that the bending moment increases along the length and the breadth of the rectangular plate, but sudden change in the bending moment decreases along the length.

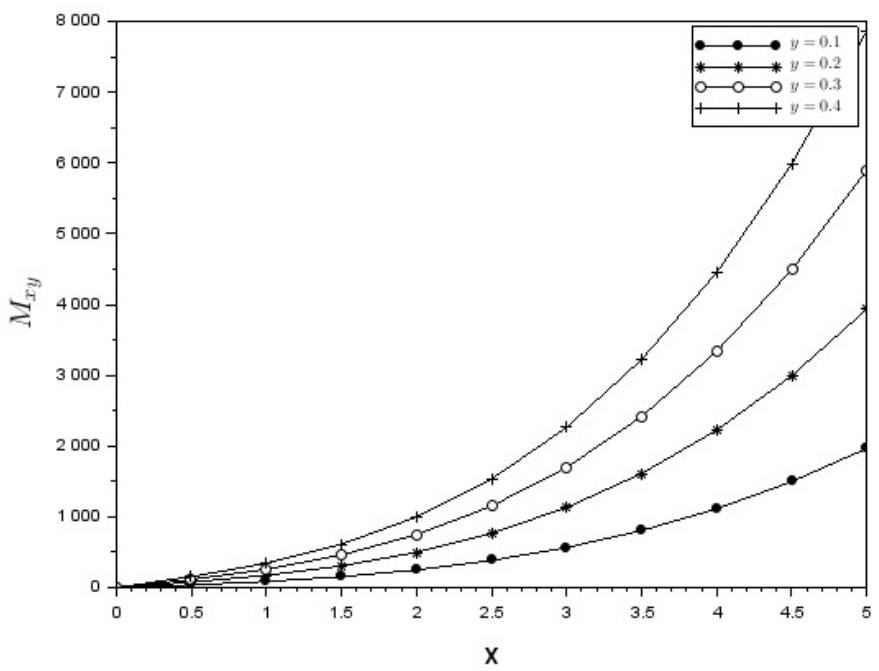

Figure 9. $M_{x y} \mathrm{Vs}^{\prime} x^{\prime}$ with variation in ' $y$ ' 
Fig-9 gives the variation of bending moments of the plate along the $z$-direction. The above figures explain the result that the bending moment increases along the length and the breadth of the rectangular plate. That means the bending moment depends on the dimensions of the rectangular plate.

\section{CONCLUSIONS}

In this paper, we consider the thick rectangular plate with simply-supported edges. The results are obtained by using the reduced differential transform method. We have determined the thermal stresses with non-homogeneous initial boundary conditions. The bending moments and thermal stresses are obtained in the form of Taylor's series. By considering the special case for the copper plate the results are discussed graphically. It is observed that the bending of the plate depends on the dimensions of the plate. The thermal stresses are also related to the dimension of the plate.

\section{REFERENCES}

1] Barletta, A. and Zanchini, E.,"Three dimensional propagation of in a solid bar with rectangular cross section hyperbolic thermal waves", International Journal of Heat and Mass Transfer,42(1999),219-229.

[2] Boley, B.A. and Weiner, J. H., Theory of Thermal Stresses, New York, 1960.

[3] Cattaneo, C., A Form of Heat Conduction Equation Which Eliminates the Paradox of Instantaneous Propagation, Compte Rendus , 247 (1958), 431-433.

[4] Chaudhari Kamini K. and Sutar C. S. , Thermoelastic modelling of a Rectangular plate under the hyperbolic heat conduction with an internal heat source, International Journal of Mechanical and Production Engineering Research and Development, (9)6 (2019), 859-872.

[5] Chen, P., Transient thermal stresses in rectangular plate due to nonuniform heat transfer coefficient, Journal of Thermal Stresses , (11)2 (1988), 115-125.

[6] Deshmukh K.C., Khandait M.V. and Kumar Rajneesh , Thermal stresses in a simply supported plate with thermal bending moments with heat sources, Materials Physics and Mechanics , 21 (2014), 135-146.

[7] Jane K.C. and Hong C.C.,Thermal bending analysis of laminated orthotropic plates by the generalized differential quadrature method, Mechanics Research Communications , (27)2 (2000), 157-164.

[8] Mallick, A.,Ranjan R.and Sarkar, P.K., Effect of heat transfer on thermal stresses in an annular hyperbolic fin: an approximate analytical solution, Journal of Theoretical and applied Mechanics , (54)2 (2016), 437-448.

[9] Mansfield E.H.Mansfield, The bending and stretching of plates (Second Edition ed.), Cambridge University Press.1989.

[10] Mehta R. C., Direct search optimization technique for the solution of inverse nonlinear heat conduction problem, Indian Journal of Engineering and Material Sciences, 19 (2012), 67-71.

[11] Mohammed O. Al-Amr, New applications of reduced differential transform method, Alexandria Engineering Journal , 53 (2014), 243-247.

[12] Morimoto, T., Tanigawa Y. and Kawamura R., Thermal bukling analysis of inhomogeneous rectangular plate due to uniform heat supply, Journal of Thermal Stresses , (26)11-12 (2003), 1151-1170.

[13] Noda, N., Hetnarski, R.B., Tanigawa, Y., Thermal Stresses, Taylor and Francis, 2003.

[14] Nowacki, W. , The state of stress in a thick circular plate due to temperature field., Bull.Sci.Acad.Polon Sci. Tech., 5 (1957).

[15] Ozicik, M., Heat Conduction (Second Edition ed.), John Wiley and Sons,Inc.,1993.

[16] Sherief, H.H and Anwar,M.N, Two-dimensional generalized thermoelasticity problem for an infinitely long cylinder, Journal of Thermal Stresses , (17)2 (1994), 213-227. 
[17] Srivastava Vineet K., Awasthi Mukesh K. and Chaurasia R.K., Reduced differential transform method to solve two and three dimensional second order hyperbolic telegraph equations, Journal of King Saud University- Engineering Sciences, 29 (2017), 166-171.

[18] Taha, B. A., The Use of Reduced Differential Transform Method for Solving Partial Differential Equations with Variable Coefficients, Journal of Basrah Researches((Sciences)), (37)4 (2011), 226-233.

[19] Timoshenko S.and Woinowsky-Krieger S., Theory of Plates and Shells (Second Edition ed.), McGraw-Hill,1989.

[20] Yos Sompornjaroensuk and Kraiwood Kiattikomol ,Bending behaviours of simply supported rectangular plates with an internal line sagged and unsagged supports, Songklanakarin Journal of Science and Technology, (30)1 (2008), 101-107.

[21] Yuemei Liu and Rui Li, Accurate bending analysis of rectangular plates with two adjacent edges free and the others clamped or simply supportedbased on new symplectic approach, Applied Mathematical Modelling, 34 (2010), 8556-865. 